\title{
Continuum description with pseudostate wave functions
}

\author{
A. M. Moro ${ }^{*}$ and M. Rodríguez-Gallardo ${ }^{\dagger}$ \\ Departamento de Física Atómica, Molecular y Nuclear, Universidad de Sevilla, Apartado 1065, E-41080 Sevilla, Spain \\ R. Crespo ${ }^{\ddagger}$ \\ Departamento de Física, Instituto Superior Técnico, Taguspark, Avenida Prof. Cavaco Silva, P-2780-990 Porto Salvo, Oeiras, Portugal \\ I. J. Thompson ${ }^{\S}$ \\ Departament of Physics, University of Surrey, Guildford GU2 7XH, United Kingdom
}

(Received 13 October 2006; published 17 January 2007)

\begin{abstract}
Benchmark calculations are performed aiming to test the use of two different pseudostate bases on the multiple scattering expansion of the total transition amplitude scattering framework. Calculated differential cross sections for $p-{ }^{6} \mathrm{He}$ inelastic scattering at $717 \mathrm{MeV} /$ nucleon show a good agreement between the observables calculated in the two bases. This result gives extra confidence on the pseudostate representation of continuum states to describe inelastic/breakup scattering.
\end{abstract}

DOI: 10.1103/PhysRevC.75.017603

PACS number(s): 24.10.-i, 24.50.+g, 25.40.Ep

Inelastic scattering at intermediate energies can be a useful tool to study multipole excitations of Borromean nuclei (such as ${ }^{11} \mathrm{Li}$ and ${ }^{6} \mathrm{He}$ ). Because of their loosely bound nature, to properly understand and interpret such reactions, it is crucial to take into account the few-body degrees of freedom. At high energies, the multiple scattering expansion of the total transition amplitude (MST) is a convenient framework that has already been applied to analyze such reactions for elastic [1,2] as well as for inelastic $[3,4]$ scattering. In the latter case, the method can take into account spin excitations that occur when scattering from a spin target such as a proton. In these calculations, it is formal and numerically advantageous to represent the continuum states in terms of a basis of square-integrable functions, also known as pseudostates (PSs). Unlike the true scattering states, the PSs vanish at large distances and hence the method will be only useful if the calculated observables are not sensitive to the asymptotic region. Moreover, calculations performed with different families of states should converge to the same results, provided that enough states are included and that the basis is complete within the radial region relevant for the process under study.

Guided by this motivation, in this Brief Report we present benchmark calculations of proton inelastic scattering from ${ }^{6} \mathrm{He}$ within the MST framework, making use of two different PS bases to describe the ${ }^{6} \mathrm{He}$ continuum. We aim to check to what extent the calculated breakup observables depend on the choice of the PS functions.

For a Borromean system, such as ${ }^{6} \mathrm{He}$, the wave function for a total angular momentum $J$ (with projection $M$ ) and energy $\epsilon, \varphi_{\epsilon}^{J M}$, can be expressed in terms of the Jacobi coordinates $\vec{r}$

\footnotetext{
*Electronic address: moro@us.es

†Electronic address: mrodri@cii.fc.ul.pt; present address: Centro de Física Nuclear, Universidade de Lisboa, Avenida Prof. Gama Pinto 2, P-1649-003 Lisboa, Portugal.

${ }^{\ddagger}$ Electronic address: Raquel.Crespo@tagus.ist.utl.pt

§Electronic address: I.Thompson@surrey.ac.uk
}

(the relative coordinate between the valence nucleons) and $\vec{R}$ (the relative coordinate from the center of mass of the neutron pair to the core).

It is also convenient to introduce a set of hyperspherical coordinates: the hyper-radius $\rho$ and five hyperspherical polar angles $\Omega_{5}=\left\{\alpha, \theta_{x}, \phi_{x}, \theta_{y}, \phi_{y}\right\}$. The former is defined as $\rho=\sqrt{x^{2}+y^{2}}$ with scaled coordinates $\vec{x}=2^{-1 / 2} \vec{r}$ and $\vec{y}=$ $(2 / \sqrt{3}) \vec{R}$. The angle $\alpha=\arctan (x / y)$ is the hyperangle and $\theta_{x}, \phi_{x}, \theta_{y}, \phi_{y}$ are the angles associated with the unit spatial vectors $\hat{x}$ and $\hat{y}$.

Within the PS method, the eigenstates $\varphi_{\epsilon}^{J M}(\vec{r}, \vec{R})$ are obtained by diagonalization of the Hamiltonian in a basis of normalizable states. These states are conveniently expanded in a basis of hyperspherical harmonics of the form

$$
\psi_{n \beta}^{J M}(\vec{r}, \vec{R})=R_{n \beta}(\rho) \Upsilon_{\beta}^{J M}\left(\Omega_{5}\right),
$$

where $\Upsilon_{\beta}^{J M}\left(\Omega_{5}\right)$ is the generalized angle-spin basis [5]

$$
\Upsilon_{\beta}^{J M}\left(\Omega_{5}\right)=\left\{\mathcal{Y}_{\mathcal{K} \ell_{x} \ell_{y} L}\left(\Omega_{5}\right) \otimes\left[\chi_{s_{2}} \otimes \chi_{s_{3}}\right]_{S}\right\}_{J M},
$$

with $\chi_{s_{i}}$ the neutron spin functions and $\mathcal{Y}_{\mathcal{K} \ell_{x} \ell_{y} L}\left(\Omega_{5}\right)$ the hyperspherical harmonics,

$$
\mathcal{Y}_{\mathcal{K} \ell_{x} \ell_{y} L M_{L}}\left(\Omega_{5}\right)=\psi_{\mathcal{K} \ell_{x} \ell_{y}}(\alpha)\left[Y_{\ell_{x}}(\hat{x}) \otimes Y_{\ell_{y}}(\hat{y})\right]_{L M_{L}} .
$$

The functions $\psi_{\mathcal{K} \ell_{x} \ell_{y}}(\alpha)$ have an explicit form in terms of Jacobi polynomials of the hyperangle $\alpha$ [5]. The set of quantum numbers $\beta=\left\{\mathcal{K} \ell_{x} \ell_{y} L S\right\}$ defines a channel, with $\ell_{x}$ and $\ell_{y}$ the orbital angular momenta associated with the Jacobi coordinates $\vec{x}$ and $\vec{y}, \mathcal{K}=\ell_{x}+\ell_{y}+2 v(v=0,1,2, \ldots)$ the hyperangular momentum, $\vec{L}=\vec{\ell}_{x}+\vec{\ell}_{y}$ the total orbital angular momentum, and $S$ the spin of the particles related by the coordinate $\vec{x}$. In Eq. (1), $R_{n \beta}(\rho)$ are the hyper-radial functions and $n$ is an index that labels the basis states within a given channel $\beta$. These functions are orthogonalized such that

$$
\int_{0}^{\infty} d \rho \rho^{5} R_{n \beta}(\rho) R_{n^{\prime} \beta}(\rho)=\delta_{n n^{\prime}} .
$$


The aim of the present work is to compare two different choices for the functions $R_{n \beta}(\rho)$ in the calculation of breakup observables within the MST framework. First, we consider the Gauss-Laguerre (GL) basis [6], whose hyper-radial part, $R_{n}^{\mathrm{GL}}(\rho)$, is given by

$$
R_{n}^{\mathrm{GL}}(\rho)=\rho_{0}{ }^{-3}[n ! /(n+5) !]^{1 / 2} L_{n}^{5}(z) \exp (-z / 2),
$$

with $z=\rho / \rho_{0}, L_{n}^{5}$ the generalized Laguerre polynomials, and $\rho_{0}$ a parameter that sets the radial scale of the basis.

The second choice is the transformed harmonic oscillator (THO) basis, recently introduced in Ref. [7] for a three-body system. The THO method is based on the idea of transforming the bound ground-state wave function of the system into the ground-state wave function of the harmonic oscillator (HO), defining a local scale transformation (LST). The ground-state wave function can be written as a linear combination of the basis functions (1),

$$
\varphi_{0}^{J_{0} M_{0}}(\vec{r}, \vec{R})=\sum_{\beta} R_{\beta}^{\epsilon_{0}}(\rho) \Upsilon_{\beta}^{J_{0} M}\left(\Omega_{5}\right),
$$

where we have introduced the abbreviated notation $\varphi_{0}^{J_{0} M_{0}} \equiv$ $\varphi_{\epsilon_{0}}^{J_{0} M_{0}}$. Then, the equation that defines the LST for each channel $\beta$ is

$$
\int_{0}^{\rho} d \rho^{\prime} \rho^{\prime 5}\left|R_{\beta}^{\epsilon_{0}}\left(\rho^{\prime}\right)\right|^{2}=\int_{0}^{s} d s^{\prime} s^{\prime 5}\left|R_{0 \mathcal{K}}^{\mathrm{HO}}\left(s^{\prime}\right)\right|^{2},
$$

where $R_{0 K}^{\mathrm{HO}}(s)$ is the hyper-radial part of the $\mathrm{HO}$ ground state for the hyperangular momentum $\mathcal{K}$. Then, the THO basis is constructed for each channel by applying the LST, $s_{\beta}(\rho)$, to the $\mathrm{HO}$ basis

$$
R_{n \beta}^{\mathrm{THO}}(\rho)=R_{\beta}^{\epsilon_{0}}(\rho) L_{n}^{\mathcal{K}+2}\left(s_{\beta}^{2}(\rho)\right),
$$

where $L_{n}^{\mathcal{K}+2}$ are generalized Laguerre polynomials of degree $n$. For channels not included in the ground state, information from one of the known (ground-state) channels with the closest quantum labels to the channel of interest is used to construct the LST, as explained in Ref. [7].

Neither the GL nor the THO functions are eigenstates of the Hamiltonian, but they provide a complete and orthonormal set in which the Hamiltonian can be diagonalized. For this purpose, the basis is truncated by setting a maximum value of the index $n(n=0, \ldots, N)$ as well as a maximum hyperangular momentum $\mathcal{K}_{\max }$. Upon diagonalization in the truncated basis, the eigenstates are obtained as

$$
\varphi_{\epsilon_{i}}^{J M}(\vec{r}, \vec{R})=\sum_{n \beta} C_{n \beta}^{J \epsilon_{i}} \psi_{n \beta}^{J M}(\vec{r}, \vec{R}),
$$

where $\left\{\epsilon_{i}\right\}$ are their associated eigenvalues.

From this derivation, it becomes apparent that the GL basis is obtained in a more straightforward way than the THO basis. However, the latter has some appealing properties that could make it more suitable in some situations. In particular, the THO basis has the advantage of being constructed from the ground-state wave function of the system. Thus, when we diagonalize the Hamiltonian in a finite THO basis, the ground state is recovered for any size of the basis. By contrast, in the GL representation a large basis may be required to obtain a good description of the ground state. Also, note that the hyper-radial part of the GL basis is the same for all the channels $\beta$ whereas in the THO basis a different hyper-radial part is calculated for each channel, with the correct behavior at the origin $\left(\rho^{\mathcal{K}}\right)$.

For a meaningful comparison between the two bases, we use the same three-body Hamiltonian to generate the GL and THO eigenstates for ${ }^{6} \mathrm{He}$. In particular, we use the $n$ - $n$ potential of Gogny, Pires, and de Tourreil [8] with spin-orbit and tensor components and we take the $n-{ }^{4} \mathrm{He}$ potential from Ref. [9]. Besides the pairwise interactions, an effective three-body potential is included, with matrix elements of the form [5]

$$
V_{\beta^{\prime} \beta}^{3 \mathrm{~B}}(\rho)=\frac{\delta_{\beta^{\prime} \beta} V_{J}^{3 \mathrm{~B}}}{1+(\rho / 5)^{3}} .
$$

The $J=0$ strength of this effective potential is tuned to reproduce the experimental three-body separation energy and the $J>0$ strength is adjusted to obtain the $2_{1}^{+}$resonance at the experimental energy.

We now consider the scattering process of ${ }^{6} \mathrm{He}$, originally in its ground state, $\left|\varphi_{0}^{J_{0} M_{0}}\right\rangle$, to a final continuum state $\left|\varphi_{\epsilon}^{J M}\right\rangle$, at excitation energy $\epsilon$ and with total angular momentum $J$ (projection $M$ ), by means of its interaction with a proton, with initial (final) linear momentum $\vec{k}_{1}\left(\vec{k}_{1}^{\prime}\right)$ in the nucleonnucleus c.m. frame and spin $S_{1}=1 / 2$ with projection $\sigma\left(\sigma^{\prime}\right)$.

The double differential cross section for this process can be formally expressed as

$$
\begin{aligned}
\frac{d^{2} \sigma_{J J_{0}}}{d \Omega d \epsilon} & =\frac{1}{\left(\widehat{S_{1}}\right)^{2}} \frac{1}{\left(\widehat{J}_{0}\right)^{2}}\left[\frac{\hbar^{2}}{4 \pi^{2} \mu_{N A}}\right]^{2} \\
& \times \sum_{\sigma \sigma^{\prime}} \sum_{M M_{0}}\left|\left\langle\vec{k}_{1}^{\prime} \chi_{S_{1}}^{\sigma^{\prime}} ; \varphi_{\epsilon}^{J M}|\mathcal{T}| \vec{k}_{1} \chi_{S_{1}}^{\sigma} ; \varphi_{0}^{J_{0} M_{0}}\right\rangle\right|^{2},
\end{aligned}
$$

where $\mathcal{T}$ denotes the transition amplitude operator [10]. This operator can be expressed as a multiple expansion series in the transition amplitudes $\hat{t}_{\mathcal{I}}$ for proton scattering from each projectile subsystem $\mathcal{I}$ [11]. At high energies and for small momentum transfers, this expansion is expected to converge quickly. If only the leading term of the series is retained, the single scattering approximation (SSA) is obtained $[3,12]$ :

$$
\mathcal{T}^{\mathrm{SSA}}=\sum_{\mathcal{I}=2}^{4} \hat{t}_{1 \mathcal{I}}
$$

with $\mathcal{I}=2,3$ for the halo neutrons, and $\mathcal{I}=4$ for the core. The proton-I subsystem transition amplitude satisfies the Lippmann-Schwinger equation

$$
\hat{t}_{1 \mathcal{I}}=v_{1 \mathcal{I}}+v_{1 \mathcal{I}} G_{0} \hat{t}_{1 \mathcal{I}},
$$

with $v_{1 \mathcal{I}}$ the interaction between the nucleon and $\mathcal{I}$ subsystem. Within the impulse approximation, the propagator $G_{0}=$ $\left(E^{+}-K\right)^{-1}$ contains the kinetic energy operators of the proton and all the projectile subsystems. Here $E$ is the kinetic energy, $E=\hbar^{2} k_{1}^{2} / 2 \mu_{N A}$ in the overall c.m. frame, and $\mu_{N A}$ is the proton-projectile reduced mass.

Within the PS method, the scattering states $\varphi_{\epsilon}^{J M}$ in Eq. (11) are approximated by the pseudostates $\varphi_{\epsilon_{i}}^{J M}$. Hence, the double differential cross section (11) becomes a single differential 
cross section for each pseudostate,

$$
\begin{aligned}
\frac{d \sigma_{J J_{0}}^{i}}{d \Omega} & =\frac{1}{\left(\widehat{S_{1}}\right)^{2}} \frac{1}{\left(\widehat{J_{0}}\right)^{2}}\left[\frac{\hbar^{2}}{4 \pi^{2} \mu_{N A}}\right]^{2} \\
& \times \sum_{\sigma \sigma^{\prime}} \sum_{M M_{0}}\left|\left\langle\vec{k}_{1}^{\prime} \chi_{S_{1}}^{\sigma^{\prime}} ; \varphi_{\epsilon_{i}}^{J M}\left|\sum_{\mathcal{I}=2}^{4} \hat{t}_{1 \mathcal{I}}\right| \vec{k}_{1} \chi_{S_{1}}^{\sigma} ; \varphi_{0}^{J_{0} M_{0}}\right\rangle\right|^{2},
\end{aligned}
$$

where we have replaced the $\mathcal{T}$ matrix operator by its single scattering approximation. By making use of the impulse approximation [2], the matrix elements for the scattering for each constituent can be further simplified, leading to the following factorized form for the scattering from one valence nucleon $(\mathcal{I}=2)$ :

$$
\begin{aligned}
& \left\langle\vec{k}_{1}^{\prime} \chi_{S_{1}}^{\sigma^{\prime}} ; \varphi_{\epsilon_{i}}^{J M}\left|\hat{t}_{12}\right| \vec{k}_{1} \chi_{S_{1}}^{\sigma} ; \varphi_{0}^{J_{0} M_{0}}\right\rangle \\
& \quad=\sum_{b \beta} t_{\left[b \beta \mathcal{S}_{p} \mathcal{S}_{p}^{\prime}\right]}\left(\omega_{12}, \Delta\right) \times \rho_{\left[b \beta ; \mathcal{S}_{T} \mathcal{S}_{T}^{\prime} \epsilon_{i}\right]}\left(\frac{m_{3}}{M_{23}} \vec{\Delta}, \frac{m_{4}}{M_{234}} \vec{\Delta}\right),
\end{aligned}
$$

with $M_{23}=m_{2}+m_{3}$ and $M_{234}=m_{2}+m_{3}+m_{4}$ and where we have introduced the momentum transfer $\vec{\Delta}=\vec{k}_{1}^{\prime}-\vec{k}_{1}$ and the energy parameter $\omega_{12}$ [2] and where $\mathcal{S}_{p}=\left\{S_{1} \sigma\right\}\left(\mathcal{S}_{p}^{\prime}=\right.$ $\left\{S_{1} \sigma^{\prime}\right\}$ ) are the incoming (outgoing) spin of the nucleon and its projection and $\mathcal{S}_{T}=\left\{J_{0} M_{0}\right\}\left(\mathcal{S}_{T}^{\prime}=\{J M\}\right)$ the initial (final) total spin of the halo valence pair. The amplitude $t_{\left[b \beta \mathcal{S}_{p} \mathcal{S}_{p}^{\prime}\right]}$ is given in terms of the tensor components of the nucleon-nucleon transition amplitude $[2,13]$. The transition

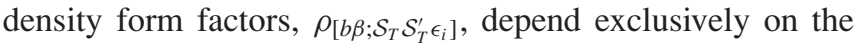
structure of the composite system. Its explicit expression as a function of the hyper-radial parts of the wave functions of the initial and final states can be found in Ref. [2].

The scattering from the core, assumed here as spinless, can equivalently be written as

$$
\begin{aligned}
& \left\langle\vec{k}_{1}^{\prime} \chi_{S_{1}}^{\sigma^{\prime}} ; \varphi_{\epsilon_{i}}^{J M}\left|\hat{t}_{14}\right| \vec{k}_{1} \chi_{S_{1}}^{\sigma} ; \varphi_{0}^{J_{0} M_{0}}\right\rangle \\
& \quad=t_{\left[00 \mathcal{S}_{p} \mathcal{S}_{p}^{\prime}\right]}\left(\omega_{14}, \Delta\right) \times \rho_{\left[00 ; \mathcal{S}_{T} \mathcal{S}_{T}^{\prime} \epsilon_{i}\right]}\left(0, \frac{M_{23}}{M_{234}} \vec{\Delta}\right),
\end{aligned}
$$

where, as before, $\omega_{14}$ is the appropriate energy parameter [2]. The angular differential cross section for ${ }^{6} \mathrm{He}$ inelastic scattering (breakup) is then obtained by summing all excitedstate contributions,

$$
\frac{d \sigma_{J J_{0}}^{\text {inel }}}{d \Omega}=\sum_{\epsilon_{i}}^{\epsilon_{i}^{\max }} \frac{d \sigma_{J J_{0}}^{i}}{d \Omega} .
$$

For the evaluation of Eqs. (15) and (16) one needs the (free) transition amplitudes for proton scattering from the valence nucleons and the core. For the former, we used the $N N$ Paris interaction. The transition amplitude for the $\alpha$ core was generated from a phenomenological optical potential, of Woods Saxon form, with parameters obtained by fitting existing data for the elastic scattering of $p+{ }^{4} \mathrm{He}$ at $E_{p}=700$ and $800 \mathrm{MeV}$, as detailed in Ref. [2].

We first study the convergence of the breakup observables with respect to the basis size. For this purpose, we consider the THO basis, truncated at different values of $N$. The maximum

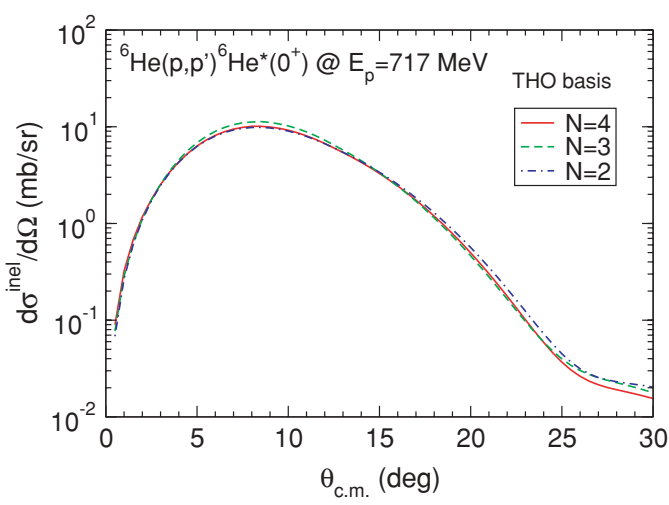

FIG. 1. (Color online) Angular differential cross section for the breakup of ${ }^{6} \mathrm{He}$ on protons at $717 \mathrm{MeV}$ per nucleon, leading to $J^{\pi}=$ $0^{+}$continuum states of the ${ }^{6} \mathrm{He}$ nucleus. The three lines represent the calculation with the THO basis, for several values of $N$ (indicated by the labels). Each curve includes the contribution from eigeinstates up to $10 \mathrm{MeV}$ in excitation energy, according to Eq. (17).

hyperangular momentum was set to $\mathcal{K}_{\max }=20$. This yields the three-body force parameters $V_{0}^{3 \mathrm{~B}}=-2.4 \mathrm{MeV}$, for $J=0$, and $V_{J}^{3 \mathrm{~B}}=-0.85 \mathrm{MeV}$, for $J>0$.

In Fig. 1 we show the angular distribution of the calculated inelastic differential cross sections. For simplicity, only the $J^{\pi}=0^{+}$continuum is included, and the Coulomb interaction between the proton and the $\alpha$ core is ignored. The three lines represent the SSA calculation for different values of the basis size, according to the choice of the parameter $N$. The three cases are in almost perfect agreement, indicating that in this reaction the convergence with the basis size is very fast.

Next, we study the dependence of the breakup observables on the choice of the basis, by comparing the calculations in the GL and THO representations. As before, the maximum hyperangular momentum was set to $\mathcal{K}_{\text {max }}=20$, and only eigenstates below $10 \mathrm{MeV}$ are considered. The index $n$ was truncated to $N=20$ and $N=4$ for the GL and THO bases, respectively. With this model space, the number of pseudostates in the GL (THO) basis is $31(30)$ for $0^{+}, 63(86)$ for $1^{-}, 53(49)$ for $1^{+}$, and $79(81)$ for $2^{+}$. For the GL basis, the range parameter was set to $\rho_{0}=0.25 \mathrm{fm}$, which provides a basis that extends up to about $20 \mathrm{fm}$ in the hyper-radius. With these parameters, the ground state obtained after diagonalization of the Hamiltonian appears at -0.9781 and $-0.9549 \mathrm{MeV}$, for the GL and THO bases, respectively.

In Fig. 2 we compare the inelastic angular distributions calculated in the GL and THO bases. The separate contributions for $J^{\pi}=0^{+}, 1^{-}, 1^{+}$, and $2^{+}$final states are also shown. As before, the Coulomb interaction is neglected. The thick lines are the incoherent sum of all these $J^{\pi}$ contributions. Solid and dashed lines correspond, respectively, to the calculations with the GL and THO bases. For each curve, the contribution of eigenstates up to $\epsilon_{\max }=10 \mathrm{MeV}$ are added incoherently, according to Eq. (17). We consider only the forward angles $\theta_{\text {c.m. }}<30^{\circ}$ since the SSA is not expected to work well at large momentum transfers [2]. We see that, at these angles, the dominant contribution to the breakup cross section comes from the $1^{-}$states, whereas for $\theta_{\text {c.m. }}>25^{\circ}$, 


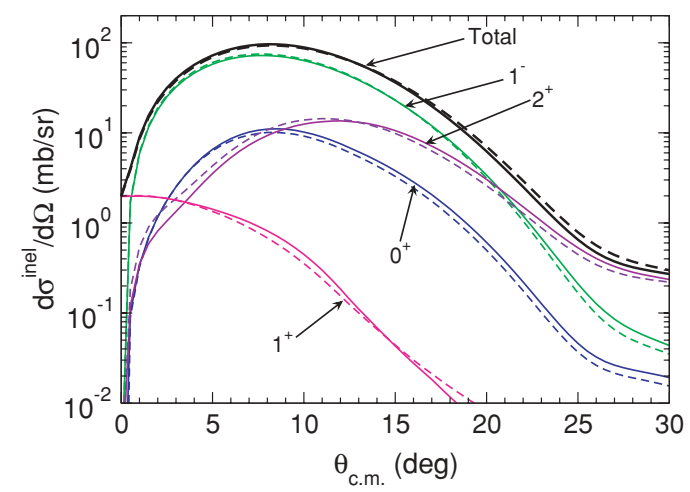

FIG. 2. (Color online) Calculated contributions for breakup differential cross section leading to final states with $J^{\pi}=0^{+}, 1^{-}, 1^{+}$, and $2^{+}$, using the SSA. Solid and dashed lines refer to the GL and THO basis, respectively.

the $2^{+}$excitation becomes dominant. Finally, the population of the unnatural parity $1^{+}$states is almost negligible at all angles. We notice that this excitation mode requires spin-flip transitions, which, according to these calculations, are very small in this reaction.

For all these contributions, the GL and THO bases provide very similar results, suggesting that the calculated observables do not depend on the choice of the continuum representation, provided that enough states are included.

In summary, in this Brief Report we have calculated proton inelastic scattering from ${ }^{6} \mathrm{He}$ at $717 \mathrm{MeV} /$ nucleon, using as scattering framework the single-scattering approximation and two different pseudostate representations of the ${ }^{6} \mathrm{He}$ continuum: the GL and the THO. Provided that enough states are included, both bases predict essentially the same inelastic differential cross section. Furthermore, the studied observables converge very quickly with the size of the basis. These results support the reliability of the pseudostate method as a useful and convenient tool to treat scattering problems dealing with continuum states. This analysis could be extended to other PS bases and reactions. Furthermore, it could be applied to other scattering frameworks, for which the PS method has also been implemented, such as the continuum discretized coupled channels method $[14,15]$.

We are grateful to J. Gómez-Camacho and J. M. Arias for useful discussions. This work was supported by the Fundação para a Ciência e Tecnologia (Portugal) through Grant No. POCTI/1999/FIS/36282, by the Acción Integrada HP20030121, and in the U.K. by EPSRC Grant No. GR/M/82141. A.M.M. acknowledges a research grant by the Junta de Andalucía.
[1] R. Crespo and I. J. Thompson, Phys. Rev. C 63, 044003 (2001).

[2] R. Crespo, A. M. Moro, and I. J. Thompson, Nucl. Phys. A771, 26 (2006).

[3] R. Crespo, I. J. Thompson, and A. A. Korsheninnikov, Phys. Rev. C 66, 021002(R) (2002).

[4] R. Crespo, I. J. Thompson, and A. M. Moro, Phys. Rev. C 74, 044616 (2006).

[5] B. V. Danilin, I. J. Thompson, M. V. Zhukov, and J. S. Vaagen, Nucl. Phys. A632, 383 (1998).

[6] I. Thompson, F. Nunes, and B. Danilin, Comput. Phys. Commun. 161, 87 (2004).

[7] M. Rodríguez-Gallardo, J. M. Arias, J. Gómez-Camacho, A. M. Moro, I. J. Thompson, and J. A. Tostevin, Phys. Rev. C 72, 024007 (2005).
[8] D. Gogny, P. Pires, and R. de Tourreil, Phys. Lett. B32, 591 (1970).

[9] J. Bang and C. Gignoux, Nucl. Phys. A313, 119 (1979).

[10] C. J. Joachain, Quantum Collision Theory (North-Holland, Amsterdam, 1987).

[11] K. M. Watson, Phys. Rev. 105, 1388 (1957).

[12] R. Crespo and R. C. Johnson, Phys. Rev. C 60, 034007 (1999).

[13] R. Crespo and A. M. Moro, Phys. Rev. C 65, 054001 (2002).

[14] A. M. Moro, F. Pérez-Bernal, J. M. Arias, and J. GómezCamacho, Phys. Rev. C 73, 044612 (2006).

[15] T. Matsumoto, E. Hiyama, K. Ogata, Y. Iseri, M. Kamimura, S. Chiba, and M. Yahiro, Phys. Rev. C 70, 061601(R) (2004). 